\title{
THE ENTROPY OF HOLOMORPHIC CORRESPONDENCES: EXACT COMPUTATIONS AND RATIONAL SEMIGROUPS
}

\author{
Gautam Bharali and Shrihari Sridharan \\ Indian Institute of Science, Department of Mathematics \\ Bangalore 560012, India; bharali@iisc.ac.in \\ Indian Institute of Science Education \& Research \\ Thiruvananthapuram 695551, India; shrihari@iisertvm.ac.in
}

\begin{abstract}
We study two notions of topological entropy of correspondences introduced by Friedland and Dinh-Sibony. Upper bounds are known for both. We identify a class of holomorphic correspondences whose entropy in the sense of Dinh-Sibony equals the known upper bound. This provides an exact computation of the entropy for rational semigroups. We also explore a connection between these two notions of entropy.
\end{abstract}

\section{Introduction, definitions and some results}

This paper studies certain semigroups of holomorphic maps. It is motivated, however, by two related notions of topological entropy - one of which applies to meromorphic correspondences on a compact Kähler manifold, while the other applies, more generally, to closed relations on a compact metric space. Both notions are thus applicable to holomorphic correspondences (which we shall define presently) on a compact Kähler manifold. The first notion is due to Dinh and Sibony [5] while the second, introduced much earlier, is due to Friedland [7]. In both cases, upper bounds for each type of topological entropy were given: by Friedland in [7] and by Dinh-Sibony in [5]. However, for either type of entropy, this upper bound is in general strictly greater than the actual entropy. In this work, among other things, we identify a natural class of holomorphic correspondences for which this upper bound equals the entropy of Dinh-Sibony.

We use the word "natural" because the above-mentioned correspondences turn out to be correspondences representing certain semigroups of holomorphic maps. Hence, the main results in this paper will be stated for these semigroups. To get to these results, we need some definitions.

Definition 1.1. Let $X_{1}$ and $X_{2}$ be two compact, connected complex manifolds of dimension $n$. A holomorphic correspondence from $X_{1}$ to $X_{2}$ is a formal linear combination of the form

$$
\Gamma=\sum_{1 \leq j \leq N} m_{j} \Gamma_{j}
$$

where the $m_{j}$ 's are positive integers and $\Gamma_{1}, \Gamma_{2}, \ldots, \Gamma_{N}$ are distinct irreducible complexanalytic subvarieties of $X_{1} \times X_{2}$ of pure dimension $n$ that satisfy the following conditions:

(1) for each $\Gamma_{j}$ in (1.1), $\left.\pi_{1}\right|_{\Gamma_{j}}$ and $\left.\pi_{2}\right|_{\Gamma_{j}}$ are surjective;

https://doi.org/10.5186/aasfm.2021.4667

2020 Mathematics Subject Classification: Primary 30D05, 37B40, 37F05; Secondary 32H50.

Key words: Holomorphic correspondences, rational semigroups, topological entropy. 
(2) for each $x \in X_{1}$ and $y \in X_{2},\left(\pi_{1}^{-1}\{x\} \cap \Gamma_{j}\right)$ and $\left(\pi_{2}^{-1}\{y\} \cap \Gamma_{j}\right)$ are finite sets for each $j$;

where $\pi_{i}$ is the projection onto $X_{i}, i=1,2$.

Given a holomorphic correspondence $\Gamma$ from $X_{1}$ to $X_{2}$, the set (in terms of the notation in (1.1)) $\bigcup_{1 \leq j \leq N} \Gamma_{j}$ is called the support of $\Gamma$, which we denote by $|\Gamma|$. The data $\left(m_{1}, \ldots, m_{N}\right)$ in $(1.1)$ are an essential part of the definition above. We shall elaborate on this below, but a brief reason is as follows. If $X_{1}=X_{2}=X$ in Definition 1.1, then we say that $\Gamma$ is a holomorphic correspondence on $X$. Two holomorphic correspondences on $X$ can be composed with each other. It is possible for a correspondence $\Gamma$, even if $m_{1}=\cdots=m_{N}=1$, to be such that some of the irreducible components of $\Gamma \circ \Gamma$ occur with multiplicity higher than 1 . The ability to compose two correspondences introduces the perspective of dynamics to the study of correspondences.

We now introduce the two notions of entropy that we alluded to. We begin with the more general notion.

Definition 1.2. (Friedland $[7,8]$ ) Let $X$ be a compact metric space and let $\Gamma$ be a closed relation on $X$ (i.e., $\Gamma$ is a closed subset of $X \times X$ and the projection $\left.\pi_{1}\right|_{\Gamma}$ is surjective). Let

$$
X^{\mathbf{N}}:=\left\{\left(x_{0}, x_{1}, x_{2}, \ldots\right): x_{n} \in X, n \in \mathbf{N}\right\}
$$

endowed with the product topology, and let

$$
\Gamma^{\infty}:=\left\{\left(x_{0}, x_{1}, x_{2}, \ldots\right) \in X^{\mathbf{N}}:\left(x_{n}, x_{n+1}\right) \in \Gamma \quad \forall n \in \mathbf{N}\right\} .
$$

If $\Gamma^{\infty}$ is endowed with the topology that it inherits from $X^{\mathbf{N}}$, then, by definition, the left-shift on $X^{\mathbf{N}}$ induces a continuous map $\sigma: \Gamma^{\infty} \longrightarrow \Gamma^{\infty}$, where $\sigma:\left(x_{0}, x_{1}, x_{2}, \ldots\right)$ $\longmapsto\left(x_{1}, x_{2}, x_{3}, \ldots\right)$. Then Friedland's entropy for $\Gamma$, denoted by $h_{F}(\Gamma)$, is defined as the topological entropy, in the sense of Bowen, of $\sigma: \Gamma^{\infty} \longrightarrow \Gamma^{\infty}$ (usually denoted by $h(\sigma)$ in the literature).

For the sake of completeness we will define the topological entropy in the sense of Bowen - see Section 3, where we examine the latter notion more closely. For the next definition, we need an alternative presentation of the correspondence introduced in Definition 1.1. We rewrite $\Gamma$ as

$$
\Gamma=\sum_{1 \leq j \leq M}^{\prime} \Gamma_{j}^{\bullet}
$$

where the primed sum indicates that the irreducible subvarieties $\Gamma_{j}^{\bullet}, j=1, \ldots, M$, are not necessarily distinct and are repeated according to multiplicity that is given by the coefficients $m_{1}, \ldots, m_{N}$ in (1.1). Therefore, $M=m_{1}+\cdots+m_{N}$. With this explanation, we give

Definition 1.3. (Dinh-Sibony [5]) Let $X$ be compact, connected complex manifold and let $\Gamma$ be a holomorphic correspondence on $X$. For each $\nu \in \mathbf{Z}_{+}$, a $\nu$-orbit of $\Gamma$ is any tuple of the form

$$
\left(x_{0}, x_{1}, \ldots, x_{\nu} ; \alpha_{1}, \ldots, \alpha_{\nu}\right) \in X^{\nu+1} \times\{1, \ldots, M\}^{\nu},
$$

where $\left(x_{j-1}, x_{j}\right) \in \Gamma_{\alpha_{j}}^{\bullet}, j=1, \ldots, \nu$, assuming the presentation (1.2) for $\Gamma$. Fix a metric $d$ compatible with the topology of $X$. If $\mathscr{F}$ is a family of $\nu$-orbits, we say that $\mathscr{F}$ is an $(\varepsilon, \nu)$-separated family, $\varepsilon>0$, if for all pairs of distinct elements

$$
\left(x_{0}, x_{1}, \ldots, x_{\nu} ; \alpha_{1}, \ldots, \alpha_{\nu}\right) \text { and }\left(y_{0}, y_{1}, \ldots, y_{\nu} ; \beta_{1}, \ldots, \beta_{\nu}\right)
$$


of $\mathscr{F}$, we have

$$
d\left(x_{j}, y_{j}\right)>\varepsilon \text { for some } j=0,1, \ldots, \nu \quad \text { or } \quad \alpha_{j} \neq \beta_{j} \quad \text { for some } j=1, \ldots, \nu .
$$

Then, the topological entropy of $\Gamma$, denoted by $h_{\text {top }}(\Gamma)$, is defined as

$$
h_{\text {top }}(\Gamma):=\sup _{\varepsilon>0} \limsup _{\nu \rightarrow \infty} \frac{1}{\nu} \log (\max \{\sharp \mathscr{F}: \mathscr{F} \text { is an }(\varepsilon, \nu) \text {-separated family }\}) \text {. }
$$

Remark 1.4. Since the manifold $X$ is compact, for any $\varepsilon>0$ and $\nu \in \mathbf{Z}_{+}$, any $(\varepsilon, \nu)$-separated family in the above definition is finite. Furthermore, it is routine to verify (see $[5$, Section 4$]$ ) that $h_{\text {top }}(\Gamma)$ does not depend on the choice of the metric $d$ for defining $(\varepsilon, \nu)$-separatedness.

Comparing the above definition with Definition 3.2, we see that Definition 1.3 is closely related to Bowen's definition of the topological entropy of maps.

Definition 1.5. A rational semigroup is a semigroup, with composition of maps as the semigroup operation, whose elements are surjective holomorphic self-maps of $\mathbf{P}^{n}$ for some $n \in \mathbf{Z}_{+}$.

Remark 1.6. Note that, despite the word "rational" in Definition 1.5, the elements of a rational semigroup on $\mathbf{P}^{n}, n \geq 2$, do not possess indeterminacies. We have some results about classical rational semigroups (i.e., defined on $(\mathbf{C} \cup\{\infty\}) \cong \mathbf{P}^{1}$ ), and do not want to coin new terminology for theorems that also hold true for higherdimensional analogues of the latter semigroups. This is the reason for the term "rational semigroups" introduced in Definition 1.5.

There is a very natural connection between finitely generated rational semigroups and holomorphic correspondences. Since this association defines the holomorphic correspondences for which we shall make exact entropy computations, let us state it formally. This association makes sense in greater generality, and not just for rational semigroups.

Definition 1.7. Let $X$ be a compact, connected complex manifold and let $S$ be a finitely generated semigroup consisting of surjective holomorphic self-maps of $X$. Let $\mathscr{G}=\left\{f_{1}, \ldots, f_{N}\right\}$ be a set of generators of $S$. We call the holomorphic correspondence

$$
\Gamma_{\mathscr{G}}:=\sum_{1 \leq j \leq N} \operatorname{graph}\left(f_{j}\right)
$$

on $X$ the holomorphic correspondence associated with $(S, \mathscr{G})$.

As has been observed earlier - see [10] by Ghys, Langevin and Walczak for the case of pseudogroups, or [8] - the entropy of a finitely-generated semigroup requires the specification of a set of generators. Thus, for $X$ and $S$ as in Definition 1.7, and for a choice $\mathscr{G}$ of a set of generators of $S$, we formally set:

$$
h_{F}(S, \mathscr{G}):=h_{F}\left(\left|\Gamma_{\mathscr{G}}\right|\right) \quad \text { and } \quad h_{\mathrm{top}}(S, \mathscr{G}):=h_{\mathrm{top}}\left(\Gamma_{\mathscr{G}}\right) .
$$

We must mention that with $S$ as above, the definition of Friedland's entropy for $S$ in [8], denoted by $h_{F}(S)$, is independent of the choice of the set of generators. Its definition is

$$
h_{F}(S):=\inf \left\{h_{F}(S, \mathscr{G}): \mathscr{G} \text { is a finite set of generators of } S\right\} .
$$

Given this definition, it is essential to first understand the quantity $h_{F}(S, \mathscr{G})$ for a given set of generators $\mathscr{G}$ - which is why we focus on $h_{F}(S, \mathscr{G})$ and $h_{\text {top }}(S, \mathscr{G})$ in this work. 
Recall that if $f: \mathbf{P}^{n} \longrightarrow \mathbf{P}^{n}$ is holomorphic, then using homogeneous coordinates, we have:

$$
f\left(\left[z_{0}: z_{1}: \cdots: z_{n}\right]\right)=\left[\mathrm{f}_{0}\left(z_{0}, z_{1}, \ldots, z_{n}\right): \mathrm{f}_{1}\left(z_{0}, z_{1}, \ldots, z_{n}\right): \cdots: \mathrm{f}_{n}\left(z_{0}, z_{1}, \ldots, z_{n}\right)\right],
$$

where there exists a number $d_{1}(f) \in \mathbf{Z}_{+}$, and $\mathbf{f}_{0}, \mathrm{f}_{1}, \ldots, \mathrm{f}_{n}$ are homogeneous polynomials of degree $d_{1}(f)$ such that $\cap_{i=0}^{n} \mathrm{f}_{i}^{-1}\{0\}=\{0\}$. With this definition, we can state our first result.

Theorem 1.8. Let $S$ be a finitely generated rational semigroup on $\mathbf{P}^{n}$ for some $n \in \mathbf{Z}_{+}$. Let $\mathscr{G}=\left\{f_{1}, \ldots, f_{N}\right\}$ be a set of generators of $S$. Then

$$
h_{\text {top }}(S, \mathscr{G})=\log \left(\sum_{1 \leq j \leq N} d_{1}\left(f_{j}\right)^{n}\right) .
$$

We can say a lot more than Theorem 1.8. The latter is a consequence of a more general theorem, which provides bounds from above and below on $h_{\text {top }}(S, \mathscr{G})$ in a more general context - see Theorem 4.2 below. Here, however: as the notion of a rational semigroup first arose in the area of complex dynamics in one dimension (see [12] by Hinkkanen and Martin) - and to foreshadow Theorem 5.2 - we state the following special case:

Corollary 1.9. Let $S$ be a finitely generated rational semigroup on $\mathbf{P}^{1}$, and let $\mathscr{G}=\left\{f_{1}, \ldots, f_{N}\right\}$ be a set of generators of $S$. Then

$$
h_{\text {top }}(S, \mathscr{G})=\log \left(\sum_{1 \leq j \leq N} \operatorname{deg}\left(f_{j}\right)\right) .
$$

We now turn to Friedland's entropy. Although it makes sense in a much more general setting, $h_{F}$ turns out to be harder to compute. This is because, among other reasons, notions that approximate concepts such as irreducible components, etc., are much less well-structured outside the complex-analytic setting, and do not feature in Definition 1.2. In the complex-analytic setting, this absence leads to two difficulties that one can point to (with $X$ here as in Definition 1.1):

(i) A holomorphic correspondence $\Gamma$ on $X$ can be iterated. If $d_{\text {top }}(\Gamma)$ denotes the topological degree of $\Gamma$ (see Section 2), then one has the identity $d_{\text {top }}\left(\Gamma^{\circ \nu}\right)=$ $d_{\text {top }}(\Gamma)^{\nu}$ for any $\nu \in \mathbf{Z}_{+}$. The analogous identity for the $\nu$-fold iterate of the relation $|\Gamma|$ - which is relevant to the entropy $h_{F}$-is not true in general. This vitiates computations of $h_{F}$.

(ii) If $X$ is Kähler, then either type of entropy is dominated by the quantity $\operatorname{lov}(\Gamma)$ (see Section 2) - which results from a technique of Gromov [11]. For similar reasons as in (i), $\operatorname{lov}(\Gamma)$ turns out not to be the best upper bound for $h_{F}(\Gamma)$ even for $\Gamma=\Gamma_{\mathscr{G}}$.

We shall elaborate upon these points and discuss further the relationship between the above notions of entropy in Section 1.1 below. For the moment, we note that rather few examples of exact computations of $h_{F}$ (not necessarily in the holomorphic category) are known: see, for instance, [8, Section 5] and [9] by Geller and Pollicott. But, as indicated above, computing $h_{F}$ is inherently hard. However, certain lower bounds for $h_{F}(S, \mathscr{G}), \mathscr{G}$ finite, are almost immediate: $h_{F}(S, \mathscr{G}) \geq h_{F}\left(\left\langle f: f \in \mathscr{G}^{\prime}\right\rangle, \mathscr{G}^{\prime}\right)$ for any $\varnothing \neq \mathscr{G}^{\prime} \mp \mathscr{G}$ (see Section 5 for details). In contrast to this, for $S$ a rational semigroup on $\mathbf{P}^{1}$, we shall establish a lower bound for $h_{F}(S, \mathscr{G})$ that takes into consideration each of the generators in $\mathscr{G}$. This is our Theorem 5.2. Since it requires some notation, we present it in Section 5. This theorem, in turn, relies on our central proposition of Section 3, which might be of independent interest. The proof of Theorem 1.8 (from which Corollary 1.9 is immediate) is presented in Section 4. 
1.1. A comparison of $\boldsymbol{h}_{\text {top }}$ and $\boldsymbol{h}_{\boldsymbol{F}}$. This section presents a bit more discussion on the two notions of entropy defined above. The material in the sections below does not depend on this discussion (and readers interested in the proofs can skip this on the first reading). The first point of contrast involves this question: given a correspondence $\Gamma$ with the presentation (1.1), is $h_{\text {top }}(\Gamma)=h_{F}(\Gamma)$ if the multiplicity of each $\Gamma_{j}$ is 1 ? The answer to this is "No", in general. To understand this answer, let us consider one of the roles these multiplicities serve. Consider a finitely generated semigroup $S$ and a set of generators $\mathscr{G}:=\left\{f_{1}, \ldots, f_{N}\right\}$. If $S$ is not freely generated and suppose, for some $\nu \in \mathbf{Z}_{+} \backslash\{1\}$, there exists a relation

$$
f_{i_{\nu}} \circ \cdots \circ f_{i_{1}}=f_{j_{\nu}} \circ \cdots \circ f_{j_{1}}=: \Phi
$$

where $\left(i_{1}, \ldots, i_{\nu}\right) \neq\left(j_{1}, \ldots, j_{\nu}\right)$, then the irreducible component graph $(\Phi)$ occurs with multiplicity at least 2 in the correspondence $\Gamma_{\mathscr{G}}^{\circ \nu}$. Given such a semigroup and a choice, $\mathscr{G}$, of a set of generators, it is usually hard to count all the relations of the form (1.5) as $\nu \rightarrow \infty$. This, hopefully, reveals explicitly one of the reasons why $h_{F}(\Gamma)$ is difficult to compute in many cases. For any holomorphic correspondence, a coarse manifestation of the issue of multiplicities - discussed here for the correspondence $\Gamma_{\mathscr{G}}$ associated with a rational semigroup - is the phenomenon mentioned in (i) above. Now, to return to the question above, the following example illustrates our answer. Let $S$ be the rational semigroup generated by $\mathscr{G}$, let $\mathscr{G}_{1}=\left\{f_{1}, f_{2}\right\}$, where $f_{1}$ and $f_{2}$ are distinct loxodromic Möbius transformations such that $f_{1}$ and $f_{2}$ have the same attracting fixed point and the same repelling fixed point. Then, while both the irreducible components of the correspondence $\Gamma_{\mathscr{G}}$ (as defined in (1.4)) occur with multiplicity 1, we have

$$
\begin{aligned}
h_{F}\left(\Gamma_{\mathscr{G}}\right) & =: h_{F}(S, \mathscr{G})=0, & & (\text { see }[8, \text { Lemma } 5.2]) \\
h_{\text {top }}\left(\Gamma_{\mathscr{G}}\right) & =: h_{\text {top }}(S, \mathscr{G})=\log (2) . & & (\text { by Corollary 1.9) }
\end{aligned}
$$

One relation between the two notions of entropy that holds universally is as follows: with $X$ as above and for any holomorphic correspondence $\Gamma$ on $X, h_{F}(\Gamma) \leq h_{\text {top }}(\Gamma)$. This is immediate from Proposition 3.5 below.

Now, $h_{\text {top }}$ has some features that may seem anomalous. E.g., with $X$ as above, if $\Gamma$ is an irreducible complex-analytic subvariety of $X \times X$ with the properties stated in Definition 1.1, then $h_{\text {top }}(2 \Gamma) \neq h_{\text {top }}(\Gamma)$. When $X=\mathbf{P}^{n}$ and $\Gamma$ is the graph of a non-constant holomorphic map $f: \mathbf{P}^{n} \longrightarrow \mathbf{P}^{n}$, then it follows from the proof of Theorem 1.8 that $h_{\text {top }}(2 \Gamma)=\log (2)+h_{\text {top }}(\Gamma)$. But this value isn't necessarily anomalous in a context where one must consider $2 \Gamma$ (instead of $\Gamma$ ). As a lot of the formalism of this paper is that of general holomorphic correspondences, one may ask why one cares for the entropy of holomorphic correspondences. It would be natural to study the entropy of these objects if one cares about the iterative dynamics of correspondences. Correspondences serve as a common framework - as the theorems above and the discussion in Section 0 of [8] testify - for a number of dynamical systems of interest. The iteration of true holomorphic correspondences on $\mathbf{P}^{1}$ (i.e., not maps) are of interest too: they realise matings between certain rational maps and certain classes of Kleinian groups: see [2,3], for instance.

\section{Complex geometry preliminaries}

This section is devoted to a discussion of terminology from geometry appearing in Section 1 whose definitions had been deferred, and to stating a result that constitutes one part of the proofs of Theorem 1.8 and Corollary 1.9. 
We first begin with a discussion of the composition of two holomorphic correspondences. Since one needs to understand this only to define a certain finite sequence of numbers associated to a correspondence $\Gamma$, we shall be brief. We refer the reader to [5, Section 3] for details (with a note to those unfamiliar with holomorphic correspondences that the footnote to [5, Section 3] is irrelevant in the case of holomorphic correspondences). We focus on two points that are relevant to this article (in what follows, $\Gamma^{\circ \nu}$ will denote the $\nu^{\text {th }}$ iterated composition of $\Gamma$ ):

(i) With $X$ as in Definition 1.1, consider two holomorphic correspondences

$$
\Gamma^{1}=\sum_{1 \leq j \leq M_{1}}^{\prime} \Gamma_{1, j}^{\bullet} \quad \text { and } \quad \Gamma^{2}=\sum_{1 \leq k \leq M_{2}}^{\prime} \Gamma_{2, k}^{\bullet}
$$

on $X$, written in accordance with the presentation (1.2). The support of $\Gamma^{2} \circ \Gamma^{1}$ is just the classical composition of $\left|\Gamma^{2}\right|$ with $\left|\Gamma^{1}\right|$ as relations. Let us denote the latter composition by $\star$. If $Y_{s, j k}, s=1, \ldots, M(j, k)$, are the distinct irreducible components of $\left|\Gamma_{2, k}^{\bullet}\right| \star\left|\Gamma_{1, j}^{\bullet}\right|$, then let

$\eta_{s, j k}:=$ the number of $y$ 's, for a generic $(x, z) \in Y_{s, j k}$, such that

$$
(x, y) \in \Gamma_{1, j}^{\bullet} \&(y, z) \in \Gamma_{2, k}^{\bullet} .
$$

Then, the definition in [5, Section 3] results in the formula:

$$
\Gamma^{2} \circ \Gamma^{1}:=\sum_{1 \leq j \leq M_{1}} \sum_{1 \leq k \leq M_{2}} \sum_{1 \leq s \leq M(j, k)} \eta_{s, j k} Y_{s, j k} .
$$

(ii) For the semigroup $S$, a choice of a set of generators $\mathscr{G}$, and the correspondence $\Gamma_{\mathscr{G}}$ introduced in Definition 1.7, we have

$$
\Gamma_{\mathscr{G}}^{\circ 2}:=\Gamma_{\mathscr{G}} \circ \Gamma_{\mathscr{G}}=\sum_{1 \leq j, k \leq N} \operatorname{graph}\left(g_{j} \circ g_{k}\right) .
$$

Observe that if $S$ is not a free semigroup and if, for instance, there exists a relation of the form $g_{j_{1}} \circ g_{k_{1}}=g_{j_{2}} \circ g_{k_{2}}$ for $\left(j_{1}, k_{1}\right) \neq\left(j_{2}, k_{2}\right)$, then the irreducible variety $\operatorname{graph}\left(g_{j_{1}} \circ g_{k_{1}}\right)$ would occur with multiplicity at least 2. Observations such as the one above are the reason why the data $\left(m_{1}, \ldots, m_{N}\right)$ in $(1.1)$ are essential in defining a holomorphic correspondence.

One can pull back certain types of currents by a holomorphic correspondencesee [4, Section 3.1]. The formal prescription for the pullback (which we denote by $F_{\Gamma}^{*}$ ) of any current $T$ of bidegree $(p, p), p=0,1, \ldots, n$ (recall that $\left.\operatorname{dim}_{\mathbf{C}}(X)=n\right)$ is:

$$
F_{\Gamma}^{*}(T):=\left(\pi_{1}\right)_{*}\left(\pi_{2}^{*}(T) \wedge[\Gamma]\right)
$$

whenever the intersection $\pi_{2}^{*}(T) \wedge[\Gamma]$ makes sense. Here, $\Gamma$ detemines a current of bidimension $(n, n)$ given by the currents of integration defined by its constituent subvarieties - which we denote by $[\Gamma]$. Recall that if $X_{1}$ and $X_{2}$ are two compact, connected complex manifolds of dimensions $n_{1}$ and $n_{2}$, respectively, $\pi: X_{1} \longrightarrow X_{2}$ is a holomorphic map, and $T$ is a current on $X_{1}$ of bidegree $(p, p), \max \left(n_{1}-n_{2}, 0\right) \leq$ $p \leq n_{1}$, then the push-forward of $T$ by $\pi$ is given by

$$
\left\langle\pi_{*} T, \varphi\right\rangle:=\left\langle T, \pi^{*} \varphi\right\rangle \quad \forall\left(n_{1}-p, n_{1}-p\right) \text {-forms } \varphi \text { on } X_{2},
$$

whereby $\pi_{*} T$ is a current of bidegree $\left(n_{2}-n_{1}+p, n_{2}-n_{1}+p\right)$ on $X_{2}$ (with the understanding that if $0 \leq p<n_{1}-n_{2}$, then $\pi_{*} T \equiv 0$ for any $(p, p)$-current $\left.T\right)$. With $X_{1}, X_{2}$ and $\pi$ as before, the pullback of a current by $\pi$ is somewhat non-standard. To begin with, one can define a pullback if $\pi$ is a submersion onto $X_{2}$. If $\pi$ is not a submersion, then the pullback is defined for special classes of $(p, p)$-currents on $X_{2}$. We refer the reader to [4, Section 2.4] for the definition of the pullback of $T$ by $\pi$ in 
these various cases. In (2.1), the map $\pi_{2}$ is a submersion and is simple enough that we have

$$
\left\langle\pi_{2}^{*}(T), \varphi\right\rangle:=\left\langle T, \int_{x \in X} \varphi(x, \cdot)\right\rangle \quad \forall(2 n-p, 2 n-p) \text {-forms } \varphi \text { on } X \times X,
$$

for a $(p, p)$-current $T, 0 \leq p \leq n$.

We consider an example where the intersection of currents in (2.1) makes sense. Any smooth $(p, p)$-form $\Theta$ on $X, p=0,1, \ldots, n$, can be pulled back by $\Gamma$ to give a $(p, p)$-current (equivalenty, a current of bidimension $(n-p, n-p))$ as follows:

$$
\left\langle F_{\Gamma}^{*}(\Theta), \varphi\right\rangle:=\sum_{j=1}^{N} m_{j} \int_{\operatorname{reg}\left(\Gamma_{j}\right)}\left(\left.\pi_{2}\right|_{\Gamma_{j}}\right)^{*} \Theta \wedge\left(\left.\pi_{1}\right|_{\Gamma_{j}}\right)^{*} \varphi \quad \forall(n-p, n-p) \text {-forms } \varphi,
$$

using the presentation (1.1) for $\Gamma$. Now suppose $(X, \omega)$ is a Kähler manifold and let $\omega_{X}$ denote the normalisation of $\omega$ so that $\int_{X} \omega_{X}^{n}=1$. For $p=0,1, \ldots, n$, we define the $p^{\text {th }}$ intermediate degree of $\Gamma$ by

$$
\lambda_{p}(\Gamma):=\left\langle F_{\Gamma}^{*}\left(\omega_{X}^{p}\right), \omega_{X}^{n-p}\right\rangle .
$$

It is well known that for each $p, \lambda_{p}$ is sub-multiplicative with respect to composition. Thus, the limit on the right-hand side below

$$
d_{p}(\Gamma):=\lim _{\nu \rightarrow \infty} \lambda_{p}\left(\Gamma^{\circ \nu}\right)^{1 / \nu}, \quad p=0,1, \ldots, n,
$$

exists. The number $d_{p}(\Gamma)$ is called the $p^{\text {th }}$ dynamical degree of $\Gamma$. Since the limit on the right-hand side of $(2.2)$ exists, $d_{p}\left(\Gamma^{\circ k}\right)=d_{p}(\Gamma)^{k}, p=0,1, \ldots, n$, for every $k \in \mathbf{Z}_{+}$.

With these definitions, we can state a result that we shall need in proving Theorem 1.8 and Corollary 1.9.

Result 2.1. (Paraphrasing of [5, Theorem 1.1]) Let $(X, \omega)$ be a compact Kähler manifold of dimension $n$ and let $\Gamma$ be a holomorphic correspondence on $X$. Then

$$
h_{\text {top }}(\Gamma) \leq \max _{0 \leq p \leq n} \log d_{p}(\Gamma) .
$$

We ought to mention that Dinh-Sibony establish the above bound on $h_{\text {top }}$ for the more general class of meromorphic correspondences. Furthermore, this bound is actually obtained - adapting a technique of Gromov [11] — by computing the value of $\operatorname{lov}(\Gamma)$, which dominates $h_{\text {top }}(\Gamma)$. Roughly speaking, $\operatorname{lov}(\Gamma)$ is the asymptotic rate of logarithmic growth (relative to $\nu$ ) of the volume of the space of all $\nu$-orbits.

\section{Notation and essential propositions on topological entropy}

We begin by fixing some notation that will be needed for the propositions in this section and in subsequent sections. The objects introduced here will pertain to a general holomorphic correspondence $\Gamma$, and our notation will be with reference to the presentation (1.2) of $\Gamma$.

We begin by introducing an object similar to $\Gamma^{\infty}$ of Definition 1.2. The parameter $M$ has the same meaning in the following definition as in (1.2):

${ }^{\Gamma} X:=\left\{\left(x_{0}, x_{1}, x_{2}, \ldots ; \alpha_{1}, \alpha_{2}, \ldots\right) \in X^{\mathbf{N}} \times\{1, \ldots, M\}^{\mathbf{Z}_{+}}:\left(x_{\nu-1}, x_{\nu}\right) \in \Gamma_{\alpha_{\nu}}^{\bullet} \forall \nu \in \mathbf{Z}_{+}\right\}$.

This space is endowed with the topology that it inherits from $X^{\mathbf{N}} \times\{1, \ldots, M\}^{\mathbf{z}_{+}}$ endowed with the product topology. We will denote by ${ }^{\Gamma} \mathscr{O}_{\nu}$ the space of all $\nu$-orbits, 
i.e.,

$\Gamma \mathscr{O}_{\nu}:=\left\{\left(x_{0}, \ldots x_{\nu} ; \alpha_{1}, \ldots, \alpha_{\nu}\right) \in X^{\nu+1} \times\{1, \ldots, M\}^{\nu}:\left(x_{j-1}, x_{j}\right) \in \Gamma_{\alpha_{j}}^{\bullet}, 1 \leq j \leq \nu\right\}$.

The above is endowed with the relative topology that it inherits from $X^{\nu+1} \times$ $\{1, \ldots, M\}^{\nu}$.

We shall need the following maps. By a mild abuse of notation, we shall denote by $\pi_{\text {itn }}$ either the map $\pi_{\text {itn }}:{ }^{\Gamma} X \longrightarrow X^{\mathbf{N}}$ or the map $\pi_{\text {itn }}: \Gamma^{\Gamma} \mathscr{O}_{\nu} \longrightarrow X^{\nu+1}$ that maps the relevant orbit of an iteration under $\Gamma$ to the itinerary of points in $X$ along that orbit. In other words:

$$
\begin{aligned}
\pi_{\text {itn }}:{ }^{\Gamma} X \ni\left(x_{0}, x_{1}, \ldots ; \alpha_{1}, \ldots\right) & \mapsto\left(x_{0}, x_{1}, \ldots\right) \text { or } \\
\pi_{\text {itn }}:{ }^{\Gamma} \mathscr{O}_{\nu} \ni\left(x_{0}, x_{1}, \ldots, x_{\nu} ; \alpha_{1}, \ldots, \alpha_{\nu}\right) & \mapsto\left(x_{0}, x_{1}, \ldots, x_{\nu}\right) \text { respectively, }
\end{aligned}
$$

where the precise definition of $\pi_{\text {itn }}$ will be obvious from the context. By a similar abuse of notation, we shall denote by $\pi_{\text {symb }}$ either the map $\pi_{\text {symb }}: \Gamma X \longrightarrow$ $\{1, \ldots, M\}^{\mathbf{Z}_{+}}$or the map $\pi_{\text {symb }}:{ }^{\Gamma} \mathscr{O}_{\nu} \longrightarrow\{1, \ldots, M\}^{\nu}$, defined by

$$
\begin{aligned}
\pi_{\mathrm{symb}}:{ }^{\Gamma} X \ni\left(x_{0}, x_{1}, \ldots ; \alpha_{1}, \ldots\right) & \mapsto\left(\alpha_{1}, \ldots\right) \text { or } \\
\pi_{\text {symb }}:{ }^{\Gamma} \mathscr{O}_{\nu} \ni\left(x_{0}, x_{1}, \ldots, x_{\nu} ; \alpha_{1}, \ldots, \alpha_{\nu}\right) & \mapsto\left(\alpha_{1}, \ldots, \alpha_{\nu}\right) \text { respectively. }
\end{aligned}
$$

Lastly, start: ${ }^{\Gamma} \mathscr{O}_{\nu} \longrightarrow X$ will denote the map $\left(x_{0}, x_{1}, \ldots, x_{\nu} ; \alpha_{1}, \ldots, \alpha_{\nu}\right) \mapsto x_{0}$. The need to consider sets of finite and infinite orbits (in ways similar to how the above objects are used here) has arisen earlier in the literature. E.g., formalisms similar to those given here for correspondences are seen in studying surjective holomorphic selfmaps of $\mathbf{P}^{2}$ due to the need to consider different prehistories (i.e., backward orbits) of a point $x_{0} \in \mathbf{P}^{2}$-see, e.g., [14] and [6].

We shall also need a standard result in elementary topology (also see Remark 3.4). We shall abbreviate $\sigma^{\circ j}$ as $\sigma^{j}$.

Lemma 3.1. Let $(Y, D)$ be a compact metric space and let $\widehat{D}$ denote the metric

$$
\widehat{D}(\widehat{x}, \widehat{y}):=\sup _{n \in \mathbf{N}} \frac{D\left(x_{n}, y_{n}\right)}{2^{n}},
$$

$\widehat{x}:=\left(x_{0}, x_{1}, x_{2}, \ldots\right)$ and $\widehat{y}:=\left(y_{0}, y_{1}, y_{2}, \ldots\right)$, which metrises the product topology on $Y^{\mathbf{N}}$. Let $\sigma:\left(x_{0}, x_{1}, x_{2}, \ldots\right) \longmapsto\left(x_{1}, x_{2}, x_{3}, \ldots\right)$ be the left-shift on $Y^{\mathbf{N}}$. Then:

$$
\max _{0 \leq j \leq n} \widehat{D}\left(\sigma^{j}(\widehat{x}), \sigma^{j}(\widehat{y})\right)=\sup _{j \in \mathbf{N}} \frac{D\left(x_{j}, y_{j}\right)}{2^{(j-n)+}},
$$

where $(j-n)+:=\max (j-n, 0)$.

Before stating the principal result of this section, we provide a couple of clarifications. In what follows, if $Y$ is a compact metric space and $f: Y \longrightarrow Y$ is a continuous map, then $h(f)$ will denote its topological entropy in the sense of Bowen. We shall define this in the setting of compact metric spaces, along with a remark on the broader concept introduced by Bowen in [1]. Since the phrase " $(\varepsilon, \nu)$-separated" appears in Definition 1.3 in a (slightly) different context, we shall - to avoid confusion-use wording that is slightly different from that in [1].

Definition 3.2. Let $(Y, D)$ be a compact metric space and let $f: Y \longrightarrow Y$ be a continuous map. A set $\mathscr{F}$ of orbits of $f$ of duration $n, n \in \mathbf{Z}_{+}$, is said to be an $\varepsilon$-separated set of orbits of $f$ of duration $n, \varepsilon>0$, if for all pairs of distinct orbits

$$
\left(x_{0}, x_{1}, \ldots, x_{n}\right) \text { and }\left(y_{0}, y_{1}, \ldots, y_{n}\right)
$$


of $\mathscr{F}$ (i.e., $x_{j}=f^{j}\left(x_{0}\right), y_{j}=f^{j}\left(y_{0}\right), j=1, \ldots, n$ )

$$
D\left(x_{j}, y_{j}\right)>\varepsilon \text { for some } j=0,1, \ldots, n \text {. }
$$

Let $M(\varepsilon, n)$ denote the greatest possible cardinality of any $\varepsilon$-separated set $\mathscr{F}$ of orbits of $f$ of duration $n$. Then, the topological entropy of $f$, denoted by $h(f)$, is defined as

$$
h(f):=\sup _{\varepsilon>0} \limsup _{n \rightarrow \infty} \frac{1}{n} \log M(\varepsilon, n) .
$$

Remark 3.3. In [1], Bowen gives a definition of entropy that does not require $Y$ to be compact. In that case, $f$ must be uniformly continuous relative to the metric on $Y$, and the value of Bowen's entropy of $f$ depends on this metric. But when $Y$ is compact, Bowen's entropy is independent of the metric, provided it metrises the topology on $Y$. This is the special case of the broader framework in [1] that we focus on in Definition 3.2.

Remark 3.4. In Definition 1.2, where $h_{F}(\Gamma)$ is defined as $h(\sigma)$ for the shift map $\sigma: \Gamma^{\infty} \longrightarrow \Gamma^{\infty}$, we now see that the latter is given by Definition 3.2 with $Y=\Gamma^{\infty}$ and $f=\sigma$. This, in fact, is the motivation for Lemma 3.1.

We now state and prove a result that will be needed in the proof of Theorem 5.2. This result is hinted at in [5, Section 4]. However:

- It is unclear if Proposition 3.5 follows, as alluded to in [5], from the conjugacy invariance of topological entropy (which applies to pairs of maps).

- For $\Gamma$ as in Definition 1.1, if the topological degree of $\left(\left.\pi_{1}\right|_{\Gamma_{j}}\right) \geq 2$ for any $j \in\{1, \ldots, N\}$, then it is unclear whether $\Gamma$ can at all be conjugated to a shift on ${ }^{\Gamma} X$.

While we shall apply Proposition 3.5 only to the holomorphic correspondence $\Gamma_{\mathscr{G}}$ in Section 5, it holds true for general holomorphic correspondences. It may thus be of independent interest. In view of the two points above, it seems worthwhile to state and give a direct proof of

Proposition 3.5. Let $X$ and $\Gamma$ be as in Definition 1.1. For $\varepsilon>0$ and $\nu \in \mathbf{Z}_{+}$, let

$N(\varepsilon, \nu):=$ the cardinality of any $(\varepsilon, \nu)$-separated family of $\nu$-orbits, in the sense of Definition 1.3, having the greatest possible cardinality.

Let $\mathscr{S}$ denote the restriction of the shift map

$$
\sigma:\left(x_{0}, x_{1}, x_{2}, \ldots ; \alpha_{1}, \alpha_{2}, \ldots\right) \mapsto\left(x_{1}, x_{2}, x_{3}, \ldots ; \alpha_{2}, \alpha_{3}, \ldots\right)
$$

to ${ }^{\Gamma} X$. Then,

$$
h_{\mathrm{top}}(\Gamma):=\sup _{\varepsilon>0} \limsup _{\nu \rightarrow \infty} \frac{1}{\nu} \log N(\varepsilon, \nu)=h(\mathscr{S}),
$$

where $h(\mathscr{S})$ is the entropy, in the sense of Bowen, of the continuous map $\mathscr{S}: \Gamma X \longrightarrow$ $\Gamma X$.

Proof. Let us fix a metric $d$ on the complex manifold $X$ that is compatible with the manifold topology. We choose the metric

$\Delta\left(\left(x_{0}, x_{1}, \ldots ; \alpha_{1}, \ldots\right),\left(y_{0}, y_{1}, \ldots ; \beta_{1}, \ldots\right)\right):=\max \left[\sup _{\nu \in \mathbf{N}} \frac{d\left(x_{\nu}, y_{\nu}\right)}{2^{\nu}}, \sup _{\nu \in \mathbf{N}} \frac{\boldsymbol{\delta}\left(\alpha_{\nu+1}, \beta_{\nu+1}\right)}{2^{\nu}}\right]$

(where $\boldsymbol{\delta}$ denotes the $0-1$ metric on the symbols $\{1, \ldots, M\}$ ) which metrises the topology on ${ }^{\Gamma} X$. Since we must show that $h_{\text {top }}(\Gamma)$ equals the Bowen entropy of $\mathscr{S}$, 
we introduce, for $\varepsilon>0$ and $\nu \in \mathbf{Z}_{+}$, the set

$$
\begin{aligned}
M(\varepsilon, \nu):= & \text { the cardinality of any } \varepsilon \text {-separated set of orbits of } \mathscr{S} \\
& \text { of duration } \nu \text { having the greatest possible cardinality. }
\end{aligned}
$$

Recall that any two orbits,

$$
\mathscr{O}_{1}:=\left(x_{0}, x_{1}, \ldots ; \alpha_{1}, \ldots\right) \text { and } \mathscr{O}_{2}:=\left(y_{0}, y_{1}, \ldots ; \beta_{1}, \ldots\right),
$$

belonging to any of the sets referred to in the definition of $M(\varepsilon, \nu)$ satisfy

$$
\max _{0 \leq j \leq \nu} \Delta\left(\mathscr{S}^{j}\left(\mathscr{O}_{1}\right), \mathscr{S}^{j}\left(\mathscr{O}_{2}\right)\right)>\varepsilon .
$$

Fix a $\nu \in \mathbf{Z}_{+}$. It suffices to consider $\varepsilon \in(0,1)$.

Let $S(\varepsilon, \nu) \subset \Gamma \mathscr{O}_{\nu}$ be an $(\varepsilon, \nu)$-separated family, in the sense of Definition 1.3, such that $\sharp S(\varepsilon, \nu)=N(\varepsilon, \nu)$. For each $\nu$-orbit $\mathrm{x} \in S(\varepsilon, \nu)$, let us pick a $\left(x_{0}, x_{1}, x_{2}, \ldots\right.$; $\left.\alpha_{1}, \alpha_{2} \ldots\right) \in \Gamma X$ such that

$$
\left(x_{0}, x_{1}, \ldots, x_{\nu} ; \alpha_{1}, \ldots, \alpha_{\nu}\right)=\mathbf{x},
$$

and fix it. Call the latter infinite orbit $\widetilde{x}$. Let us consider two distinct $\nu$-orbits

$$
\mathbf{x}:=\left(x_{0}, x_{1}, \ldots, x_{\nu} ; \alpha_{1}, \ldots, \alpha_{\nu}\right) \text { and } \mathbf{y}:=\left(y_{0}, y_{1}, \ldots, y_{\nu} ; \beta_{1}, \ldots, \beta_{\nu}\right)
$$

belonging to $S(\varepsilon, \nu)$. We have two possibilities for the pair $\{\mathrm{x}, \mathrm{y}\}$ :

Case 1. $\max _{0 \leq j \leq \nu} d\left(x_{j}, y_{j}\right)>\varepsilon$. Then (with the meaning of $\widetilde{y}$ hopefully being clear) by the definition of $\Delta$, and in view of Lemma 3.1, we have

$$
\max _{0 \leq j \leq \nu} \Delta\left(\mathscr{S}^{j}(\widetilde{\mathrm{x}}), \mathscr{S}^{j}(\widetilde{\mathrm{y}})\right) \geq \sup _{j \in \mathbf{N}} \frac{d\left(x_{j}, y_{j}\right)}{2^{(j-\nu)+}}>\varepsilon .
$$

Case 2. $\max _{0 \leq j \leq \nu} d\left(x_{j}, y_{j}\right) \leq \varepsilon$. In this case, by (1.3) there exists a $j^{*}$, with $1 \leq j^{*} \leq \nu$, such that $\alpha_{j^{*}} \neq \beta_{j^{*}}$. Therefore, in view of Lemma 3.1, we have

$$
\max _{0 \leq j \leq \nu} \Delta\left(\mathscr{S}^{j}(\widetilde{\mathrm{x}}), \mathscr{S}^{j}(\widetilde{\mathrm{y}})\right) \geq \sup _{j \in \mathbf{N}} \frac{\boldsymbol{\delta}\left(\alpha_{j+1}, \beta_{j+1}\right)}{2^{(j-\nu)+}} \geq 1>\varepsilon .
$$

From (3.3) and (3.4) it follows that the set $\{\widetilde{\mathrm{x}} \in \Gamma \mathscr{O}: \mathrm{x} \in S(\varepsilon, \nu)\}$ is an $\varepsilon$-separated set of orbits of $\mathscr{S}$ in the sense of (3.2). Since the latter set has cardinality $N(\varepsilon, \nu)$, we get:

$$
M(\varepsilon, \nu) \geq N(\varepsilon, \nu)
$$

Now let $\Sigma(\varepsilon, \nu) \subset \Gamma X$ be an $\varepsilon$-separated set of orbits in the sense of (3.2) such that $\sharp \Sigma(\varepsilon, \nu)=M(\varepsilon, \nu)$. Then, for two distinct orbits $\mathscr{O}_{1}, \mathscr{O}_{2} \in \Sigma(\varepsilon, \nu)$, we have (using the notation in (3.1)):

$$
\begin{aligned}
\frac{d\left(x_{j}, y_{j}\right)}{2^{(j-\nu)+}} \leq \varepsilon \quad \forall j \geq \nu+\log _{2}(1 / \varepsilon)+\log _{2}(\operatorname{diam}(X)), \\
\frac{\boldsymbol{\delta}\left(\alpha_{j+1}, \beta_{j+1}\right)}{2^{(j-\nu)+}} \leq \varepsilon \quad \forall j \geq \nu+\log _{2}(1 / \varepsilon),
\end{aligned}
$$

where $\log _{2}(t):=\log (t) / \log (2) \forall t>0$. Given the definition of the metric $\Delta$, it is impossible for the quantities

$$
\max _{0 \leq j \leq \nu} \sup _{k \in \mathbf{N}} \frac{d\left(\pi_{k} \circ \pi_{\text {itn }}\left(\mathscr{S}^{j}\left(\mathscr{O}_{1}\right)\right), \pi_{k} \circ \pi_{\text {itn }}\left(\mathscr{S}^{j}\left(\mathscr{O}_{2}\right)\right)\right)}{2^{k}},
$$


and

$$
\max _{0 \leq j \leq \nu} \sup _{k \in \mathbf{Z}_{+}} \frac{\boldsymbol{\delta}\left(\pi_{k} \circ \pi_{\mathrm{symb}}\left(\mathscr{S}^{j}\left(\mathscr{O}_{1}\right)\right), \pi_{k} \circ \pi_{\mathrm{symb}}\left(\mathscr{S}^{j}\left(\mathscr{O}_{2}\right)\right)\right)}{2^{k-1}}
$$

(where $\pi_{k}$ denotes the projection onto the $k^{\text {th }}$ factor) to both be less than or equal to $\varepsilon$. Thus, by (3.6), (3.7) and Lemma 3.1, we have

$$
d\left(x_{j}, y_{j}\right)>2^{(j-\nu)+} \varepsilon \quad \text { or } \quad \boldsymbol{\delta}\left(\alpha_{j+1}, \beta_{j+1}\right) \neq 0 \quad \text { for some } j: 0 \leq j \leq C(\varepsilon)+\nu,
$$

where $C(\varepsilon)$ is the greatest integer that is strictly less than $\log _{2}(1 / \varepsilon)+\log _{2}(\operatorname{diam}(X))$. Hence

$$
\left(x_{0}, x_{1} \ldots, x_{C(\varepsilon)+\nu} ; \alpha_{1}, \ldots, \alpha_{C(\varepsilon)+\nu}\right) \quad \text { and } \quad\left(y_{0}, y_{1} \ldots, y_{C(\varepsilon)+\nu} ; \beta_{1}, \ldots, \beta_{C(\varepsilon)+\nu}\right)
$$

are $(\varepsilon, C(\varepsilon)+\nu)$-separated in the sense of Definition 1.3. Since this applies to any pair of distinct $\mathscr{O}_{1}, \mathscr{O}_{2} \in \Sigma(\varepsilon, \nu)$, we get

$$
N(\varepsilon, C(\varepsilon)+\nu) \geq M(\varepsilon, \nu) .
$$

From this and (3.5), it follows that

$$
N(\varepsilon, \nu) \leq M(\varepsilon, \nu) \leq N(\varepsilon, C(\varepsilon)+\nu) .
$$

From the above, and from the definitions of the numbers $M(\varepsilon, \nu)$ and $N(\varepsilon, \nu)$, the result is now immediate.

We end this section revisiting Bowen's entropy. It will be needed in the proof of Theorem 5.2. To state it, we need some terminology. Let $(Y, D)$ be as in Lemma 3.1 and let $f: Y \longrightarrow Y$ be a continuous map. Let $K \subseteq Y$. Given $\varepsilon>0$ and $n \in \mathbf{Z}_{+}$, a subset $\mathscr{F} \subset Y$ is said to $(\varepsilon, n)$-span $K$ with respect to $f$ if for each $x \in K$ there exists a $y \in \mathscr{F}$ so that

$$
D\left(f^{j}(x), f^{j}(y)\right) \leq \varepsilon \quad \forall j=0,1, \ldots, n-1 .
$$

Let $r_{n}(\varepsilon, K):=\inf \{\sharp \mathscr{F}: \mathscr{F} \subset Y(\varepsilon, n)$-spans $K\}$. If $K$ is compact, then, clearly, $r_{n}(\varepsilon, K)$ is finite for any $\varepsilon>0$ and $n \in \mathbf{Z}_{+}$. Now, define:

$$
h(f, K):=\lim _{\varepsilon \rightarrow 0^{+}} \limsup _{n \rightarrow \infty} \frac{1}{n} \log \left(r_{n}(\varepsilon, K)\right) .
$$

We must admit that, in the above discussion, we are omitting a considerable amount of context. For instance, the quantity $h(f, K)$ is an ingredient in the definition of Bowen's entropy, which-as mentioned in Remark 3.3- does not require $Y$ to be compact. Before we state the result that we need, we must mention that as $Y$ above is compact, $h(f, K)$ does not depend on the choice of $D$ (provided it metrises the topology on $Y)$ : see the proof of [1, Proposition 3].

Result 3.6. (Bowen [1, Theorem 17]) Let $\left(Y_{i}, d_{i}\right), i=1,2$, be two compact metric spaces. Let $f_{i}: Y_{i} \longrightarrow Y_{i}, i=1,2$, be continuous surjective maps. Let $\pi: Y_{1} \longrightarrow Y_{2}$ be a continuous surjective map such that $\pi \circ f_{1}=f_{2} \circ \pi$. Then

$$
h\left(f_{2}\right) \leq h\left(f_{1}\right) \leq h\left(f_{2}\right)+\sup _{y \in Y_{2}} h\left(f_{1}, \pi^{-1}\{y\}\right) .
$$




\section{The proof of Theorem 1.8}

This section will chiefly be devoted to Theorem 4.2 below. Theorem 1.8 would follow as its corollary. But before we can prove Theorem 4.2, we must present an auxiliary quantity and a lemma. To do so, let $X, \Gamma$ and $d$ be as in Definition 1.3. For a given $\nu \in \mathbf{Z}_{+}$, fix a $\nu$-tuple $\overline{\boldsymbol{\alpha}}:=\left(\alpha_{1}, \ldots, \alpha_{\nu}\right) \in\{1, \ldots, M\}^{\nu}$. We say that a family $\mathscr{F}$ of $\nu$-orbits is $(\varepsilon, \overline{\boldsymbol{\alpha}})$-separated if for all pairs of distinct elements

$$
\left(x_{0}, x_{1}, \ldots, x_{\nu} ; \beta_{1}, \ldots, \beta_{\nu}\right) \text { and }\left(y_{0}, y_{1}, \ldots, y_{\nu} ; \gamma_{1}, \ldots, \gamma_{\nu}\right)
$$

of $\mathscr{F}$, we have

- $\left(\beta_{1}, \ldots, \beta_{\nu}\right)=\overline{\boldsymbol{\alpha}}=\left(\gamma_{1}, \ldots, \gamma_{\nu}\right)$; and

- $\max _{0 \leq j \leq \nu} d\left(x_{j}, y_{j}\right)>\varepsilon$.

Lemma 4.1. Let $X$ and $\Gamma$ be as in Definition 1.3. For $\varepsilon>0, \nu \in \mathbf{Z}_{+}$and $\overline{\boldsymbol{\alpha}} \in\{1, \ldots, M\}^{\nu}$, let

$$
\begin{array}{r}
n(\varepsilon, \overline{\boldsymbol{\alpha}}):=\text { the cardinality of any }(\varepsilon, \overline{\boldsymbol{\alpha}}) \text {-separated family of } \\
\\
\quad \nu \text {-orbits having the greatest possible cardinality. }
\end{array}
$$

Then,

$$
h_{\text {top }}(\Gamma)=\sup _{\varepsilon>0} \limsup _{\nu \rightarrow \infty} \frac{1}{\nu} \log \left[\sum_{\overline{\boldsymbol{\alpha}} \in\{1, \ldots, M\}^{\nu}} n(\varepsilon, \overline{\boldsymbol{\alpha}})\right] .
$$

The proof of this lemma is extremely elementary. But since it is vital to the proof of Theorem 4.2, we provide the following

Outline of proof. Fix an $\varepsilon>0$ and $\nu \in \mathbf{Z}_{+}$. For $\overline{\boldsymbol{\alpha}} \in\{1, \ldots, M\}$, let $\mathscr{F}(\overline{\boldsymbol{\alpha}})$ be an $(\varepsilon, \overline{\boldsymbol{\alpha}})$-separated family such that $\sharp \mathscr{F}(\overline{\boldsymbol{\alpha}})=n(\varepsilon, \overline{\boldsymbol{\alpha}})$. Write

$$
\mathscr{F}:=\bigcup_{\overline{\boldsymbol{\alpha}} \in\{1, \ldots, M\}^{\nu}} \mathscr{F}(\overline{\boldsymbol{\alpha}}) .
$$

The lemma follows from the fact that $\mathscr{F}$ is an $(\varepsilon, \nu)$-separated family and that $\sharp \mathscr{F}=$ $N(\varepsilon, \nu)$-where $N(\varepsilon, \nu)$ is as introduced in Proposition 3.5. Both these statements follow from the definitions and the fact that if

$$
\begin{aligned}
\left(x_{0}, x_{1}, \ldots, x_{\nu} ; \alpha_{1}, \ldots, \alpha_{\nu}\right) & \in \mathscr{F}\left(\left(\alpha_{1}, \ldots, \alpha_{\nu}\right)\right), \\
\left(y_{0}, y_{1}, \ldots, y_{\nu} ; \beta_{1}, \ldots, \beta_{\nu}\right) & \in \mathscr{F}\left(\left(\beta_{1}, \ldots, \beta_{\nu}\right)\right),
\end{aligned}
$$

and $\left(\alpha_{1}, \ldots, \alpha_{\nu}\right) \neq\left(\beta_{1}, \ldots, \beta_{\nu}\right)$, then these two $\nu$-orbits are $(\varepsilon, \nu)$-separated in the sense of Definition 1.3.

We can now present the central result of this section. In what follows, $d_{\text {top }}$ will denote the topological degree, while for a surjective holomorphic map $f: X \longrightarrow X$, $X$ a compact Kähler manifold, $d_{p}(f)$ will denote the $p^{\text {th }}$ dynamical degree of $\operatorname{graph}(f)$ (see Section 2). We must also spell out what is meant by the topological degree of a holomorphic correspondence. Let $X_{1}$ and $X_{2}$ be as in Definition 1.1 and $\Gamma$ be a holomorphic correspondence from $X_{1}$ to $X_{2}$. Representing $\Gamma$ as in (1.1), it is classical that there is a Zariski-open set $W \subset X_{2}$ and $d_{j} \in \mathbf{Z}_{+}$such that $\left(\pi_{2}^{-1}(W) \cap \Gamma_{j}, W, \pi_{2}\right)$ is a $d_{j}$-sheeted covering. The topological degree of $\Gamma$ is defined as

$$
d_{\text {top }}(\Gamma):=\sum_{1 \leq j \leq N} m_{j} d_{j}
$$

In other words, $d_{\text {top }}(\Gamma)$ is the generic number of preimages of a point counted according to multiplicity. If $X_{1}=X_{2}=X$ and $\Gamma=\operatorname{graph}(f)$, where $f: X \longrightarrow X$ is a 
surjective holomorphic map, then the latter definition applied to graph $(f)$ coincides with the classical definition of the topological degree of $f$.

One half of our proof of the following theorem is strongly influenced by the derivation by Misiurewicz and Przytycki [15] of a lower bound for topological entropy of a single map in the $\mathcal{C}^{1}$ setting. Our notation below follows the treatment of the above result by Katok-Hasselblatt in [13, Chapter 8].

Theorem 4.2. Let $(X, \omega)$ be a compact Kähler manifold of dimension $n$ and let $S$ be a finitely generated semigroup consisting of surjective holomorphic self-maps of $X$. Let $\mathscr{G}=\left\{f_{1}, \ldots, f_{N}\right\}$ be a set of generators of $S$. Then

$$
\begin{aligned}
\log \left(\sum_{j=1}^{N} d_{\text {top }}\left(f_{j}\right)\right) & \leq h_{\text {top }}(S, \mathscr{G}) \\
& \leq \max \left[\log (N), \log \left(\sum_{j=1}^{N} d_{\text {top }}\left(f_{j}\right)\right), \max _{1 \leq p \leq n-1} \log d_{p}\left(\Gamma_{\mathscr{G}}\right)\right] .
\end{aligned}
$$

Proof. Let $\omega_{X}$ be the normalisation of the form $\omega$ as in Section 2. For any holomorphic correspondence $\Gamma$ on $X$, we have (see [4, Section 3.1], for instance):

$$
\lambda_{n}(\Gamma)=\sum_{1 \leq j \leq N} m_{j} \int_{\operatorname{reg}\left(\Gamma_{j}\right)}\left(\left.\pi_{2}\right|_{\Gamma_{j}}\right)^{*} \omega_{X}
$$

assuming the presentation (1.2) for $\Gamma$. Since, for each $j=1, \ldots, N,\left(\Gamma_{j}, \pi_{2}, X\right)$ is a holomorphic branched covering, if follows from (4.2) and a change-of-variable argument that $\lambda_{n}(\Gamma)$ equals the topological degree of $\Gamma$ : call if $d_{\text {top }}(\Gamma)$. Since the toplogical degree is multiplicative with respect to composition, it follows from (2.2) that

$$
d_{n}(\Gamma)=\lim _{\nu \rightarrow \infty} d_{\mathrm{top}}\left(\Gamma^{\circ \nu}\right)^{1 / \nu}=d_{\mathrm{top}}(\Gamma)
$$

for any holomorphic correspondence $\Gamma$ on $X$. Applying this to the correspondence $\Gamma_{\mathscr{G}}$ we get

$$
d_{n}\left(\Gamma_{\mathscr{G}}\right)=\sum_{1 \leq j \leq N} d_{\mathrm{top}}\left(f_{j}\right)
$$

A completely analogous discussion (whose details we leave to the reader) gives us $d_{0}\left(\Gamma_{\mathscr{G}}\right)=N$. Recalling the definition of $h_{\text {top }}(S, \mathscr{G})$, the upper bound in (4.1) follows from the last identity, (4.3), and Result 2.1.

For any holomorphic map $f: X \longrightarrow X$, let Jac $(f)$ denote the real Jacobian of $f$ determined by the volume form $\omega^{n}$. Since $f$ is holomorphic, $\operatorname{Jac}(f) \geq 0$. Fix a metric $d$ that metrises the topology of $X$. Fix a number $L$ such that

$$
L>1 \quad \text { and } \sup _{x \in X} \operatorname{Jac}\left(f_{j}\right)(x) \leq L, \quad j=1, \ldots, N .
$$

Let us pick a number $\beta \in(0,1)$ and set $\delta(\beta):=L^{-\beta /(1-\beta)}$. Define the sets

$$
\mathcal{B}(\beta, j):=\left\{x \in X: \operatorname{Jac}\left(f_{j}\right)(x) \geq \delta(\beta)\right\}, \quad j=1, \ldots, N,
$$

and consider the open cover consisting of balls,

$$
\mathscr{C}(\beta, j):=\left\{B_{d}\left(x ; r_{x}\right): x \in \mathcal{B}(\beta, j) \text { and }\left.f_{j}\right|_{B_{d}\left(x ; r_{x}\right)} \text { is invertible }\right\},
$$

of $\mathcal{B}(\beta, j), j=1, \ldots, N$. Let $\varepsilon(\beta, j) \in(0,1)$ be a Lebesgue number of $\mathscr{C}(\beta, j)$ (each $\mathcal{B}(\beta, j)$ is compact) and write $\varepsilon(\beta):=\min _{1 \leq j \leq N} \varepsilon(\beta, j)$.

Fix a $\nu \in \mathbf{Z}_{+}$. We simplify the symbol $\Gamma_{\mathscr{G}} \mathscr{O}_{\nu}$ to $\mathscr{G}^{\mathscr{O}}{ }_{\nu}$. For each $\overline{\boldsymbol{\alpha}} \in\{1, \ldots, N\}^{\nu}$, define

$$
A_{\beta, \overline{\boldsymbol{\alpha}}}:=\left\{\left(x_{0}, x_{1}, \ldots, x_{\nu} ; \overline{\boldsymbol{\alpha}}\right) \in{ }^{\mathscr{G}} \mathscr{O}_{\nu} \mid \sharp\left\{1 \leq k \leq \nu: x_{k-1} \in \mathcal{B}\left(\beta, \alpha_{k}\right)\right\} \leq \beta \nu\right\} .
$$


For any $l: 1 \leq l \leq \nu$, let us abbreviate

$$
f_{\alpha_{l}} \circ \cdots \circ f_{\alpha_{1}}=: f_{\left(\alpha_{1}, \ldots, \alpha_{l}\right)} .
$$

Whenever $\nu \geq 2$, the chain rule gives

$$
\operatorname{Jac}\left(f_{\left(\alpha_{1}, \ldots, \alpha_{\nu}\right)}\right)(x)=\left[\prod_{2 \leq k \leq \nu} \operatorname{Jac}\left(f_{\alpha_{k}}\right)\left(f_{\left(\alpha_{1}, \ldots, \alpha_{k-1}\right)}(x)\right)\right] \operatorname{Jac}\left(f_{\alpha_{1}}\right)(x) .
$$

Therefore, by the definitions of $A_{\beta, \overline{\boldsymbol{\alpha}}}$ and $L$ (for any $\nu \in \mathbf{Z}_{+}$):

$$
\begin{aligned}
0 \leq \operatorname{Jac}\left(f_{\left(\alpha_{1}, \ldots, \alpha_{\nu}\right)}\right)\left(x_{0}\right) & <\delta(\beta)^{\nu-[\beta \nu]} L^{[\beta \nu]} \\
& \leq \delta(\beta)^{\nu(1-\beta)} L^{\beta \nu}=1 \quad \forall\left(x_{0}, x_{1}, \ldots, x_{\nu} ; \overline{\boldsymbol{\alpha}}\right) \in A_{\beta, \overline{\boldsymbol{\alpha}}},
\end{aligned}
$$

where here (and elsewhere in this proof) $[s]$ denotes the greatest integer less than or equal to $s$. If $\mu_{X}$ denotes the Borel measure, constructed in the standard manner, with the property that $\mu_{X}(\Omega):=\int_{\Omega} \omega^{n}$ for every coordinate patch $\Omega \subseteq X$, then:

(a) (4.4) implies that $\mu_{X}\left(f_{\left(\alpha_{1}, \ldots, \alpha_{\nu}\right)}\left(A_{\beta, \overline{\boldsymbol{\alpha}}}\right)\right)<\mu_{X}(X)$ for each $\overline{\boldsymbol{\alpha}}=\left(\alpha_{1}, \ldots, \alpha_{\nu}\right) \in$ $\{1, \ldots, N\}^{\nu}$.

(b) Thus, if we fix an $\overline{\boldsymbol{\alpha}}$, then, by Sard's Theorem, there exists a point in $X \backslash$ $f_{\overline{\boldsymbol{\alpha}}}\left(A_{\beta}, \overline{\boldsymbol{\alpha}}\right)$ that is a regular value of $f_{\overline{\boldsymbol{\alpha}}}$.

Let us call this regular value $x_{\nu}$.

For the $\overline{\boldsymbol{\alpha}}=\left(\alpha_{1}, \ldots, \alpha_{\nu}\right)$ fixed in (b) above, for any $\alpha_{k}, 1 \leq k \leq \nu$, and any regular value $y$ of $f_{\alpha_{k}}$, we present a construction associated with the pair $(y, k)$. Define

$$
S(y, k):= \begin{cases}f_{\alpha_{k}}^{-1}\{y\}, & \text { if } f_{\alpha_{k}}^{-1}\{y\} \subset \mathcal{B}\left(\beta, \alpha_{k}\right), \\ \left\{x^{(y)}\right\}, & \text { if } f_{\alpha_{k}}^{-1}\{y\} \not \subset \mathcal{B}\left(\beta, \alpha_{k}\right),\end{cases}
$$

where, $x^{(y)}$ denotes some point in $f_{\alpha_{k}}^{-1}\{y\} \backslash \mathcal{B}\left(\beta, \alpha_{k}\right)$ that we pick and fix. We now consider the point $x_{\nu}$ introduced at the end of the previous paragraph. We will use it to construct a certain $(\varepsilon(\beta), \overline{\boldsymbol{\alpha}})$-separated family in ${ }^{\mathscr{G}} \mathscr{O}_{\nu}$ using the following iterative construction. This construction is possible because, as every $f \in S$ is surjective, by the definition of a regular value we get:

each element of $\left(f_{\alpha_{\nu}} \circ \cdots \circ f_{\alpha_{k+1}}\right)^{-1}\left\{x_{\nu}\right\}$ is a regular value of $f_{\alpha_{k}}$ for $1 \leq k \leq \nu-1$.

Define (the maps appearing below were defined in Section 3):

$$
\begin{aligned}
{ }^{1} O_{\overline{\boldsymbol{\alpha}}} & :=\left\{\left(x, x_{\nu} ; \alpha_{\nu}\right): x \in S\left(x_{\nu}, \nu\right)\right\}, \\
{ }^{k+1} O_{\overline{\boldsymbol{\alpha}}} & :=\bigcup_{\xi \in{ }^{k} O_{\nu}}\left\{\left(x, \pi_{\mathrm{itn}}(\xi) ; \alpha_{\nu-k}, \ldots, \alpha_{\nu}\right): x \in S(\operatorname{start}(\xi), \nu-k)\right\}, \quad 1 \leq k \leq \nu-1 .
\end{aligned}
$$

Here, we commit a minor abuse of notation in that if, for $1 \leq k \leq \nu-1,{ }^{k} O_{\nu} \ni \xi=$ $\left(x_{\nu-k}, \ldots, x_{\nu} ; \alpha_{\nu-k+1}, \ldots, \alpha_{\nu}\right)$, then we interpret $\left(x, \pi_{\text {itn }}(\xi) ; \alpha_{\nu-k}, \ldots, \alpha_{\nu}\right)$ to mean

$$
\left(x, x_{\nu-k}, \ldots, x_{\nu} ; \alpha_{\nu-k}, \ldots, \alpha_{\nu}\right) \text { and } \operatorname{not}\left(x,\left(x_{\nu-k}, \ldots, x_{\nu}\right) ; \alpha_{\nu-k}, \ldots, \alpha_{\nu}\right) .
$$

With this explanation, note that each ${ }^{k} O_{\nu}$ is a collection of $k$-orbits that end at the point $x_{\nu}$. The iterative construction lengthens each $k$-orbit $\xi \in{ }^{k} O_{\nu}$ to one or more $(k+1)$-orbits by designating new initial points for the latter orbits.

Let us write $O_{\overline{\boldsymbol{\alpha}}}:={ }^{\nu} O_{\overline{\boldsymbol{\alpha}}}$. We now show that $O_{\overline{\boldsymbol{\alpha}}}$ is an $(\varepsilon(\beta), \overline{\boldsymbol{\alpha}})$-separated family of $\nu$-orbits. To do so, consider two distinct $\nu$-orbits

$$
\mathrm{x}:=\left(x_{0}, \ldots, x_{\nu-1}, x_{\nu} ; \overline{\boldsymbol{\alpha}}\right) \quad \text { and } \quad \mathrm{y}:=\left(y_{0}, \ldots, y_{\nu-1}, x_{\nu} ; \overline{\boldsymbol{\alpha}}\right)
$$


in $O_{\bar{\alpha}}$ (note that, by construction, the terminal points of these $\nu$-orbits are the same). Write

$$
\tau:=\max \left\{1 \leq k \leq \nu: x_{k-1} \neq y_{k-1}\right\} .
$$

By our iterative construction, $x_{\tau-1}, y_{\tau-1} \in f_{\alpha_{\tau}}^{-1}\left\{x_{\tau}\right\}$. In terms of the notation introduced above, this also tells us that $\sharp S\left(x_{\tau}, \tau\right) \geq 2$. This means that

$$
x_{\tau-1}, y_{\tau-1} \in \mathcal{B}\left(\beta, \alpha_{\tau}\right) \text { and } f_{\alpha_{\tau}} \text { is injective on small balls around } x_{\tau-1}, y_{\tau-1} \text {. }
$$

Clearly, $x_{\tau-1}$ and $y_{\tau-1}$ cannot belong to one single ball belonging to the open cover $\mathscr{C}\left(\beta, \alpha_{\tau}\right)$. Thus, by the definition of Lebesgue number, $d\left(x_{\tau-1}, y_{\tau-1}\right)>\varepsilon(\beta)$. Since $\mathrm{x} \neq \mathrm{y} \in O_{\overline{\boldsymbol{\alpha}}}$ were arbitrarily chosen, we conclude that $O_{\overline{\boldsymbol{\alpha}}}$ is an $(\varepsilon(\beta), \overline{\boldsymbol{\alpha}})$-separated family.

Write $d_{j}:=d_{\text {top }}\left(f_{j}\right), j=1, \ldots, N$. We may assume without loss of generality that $d_{1} \leq \cdots \leq d_{N}$. Let $\nu_{j}:=$ the number of times $j$ appears in $\overline{\boldsymbol{\alpha}}$. Let us now set

$$
m:=[\beta \nu]+1, \quad J:=\max \left\{1 \leq j \leq N: \nu_{1}+\cdots+\nu_{j}<m\right\} .
$$

By construction, for each $\nu$-orbit $\mathrm{x} \in O_{\overline{\boldsymbol{\alpha}}}$, start $(x) \in f_{\overline{\boldsymbol{\alpha}}}^{-1}\left\{x_{\nu}\right\}$. Hence, by our above choice of $x_{\nu}$,

$$
\left\{\operatorname{start}(\mathrm{x}): \mathrm{x} \in O_{\bar{\alpha}}\right\} \cap A_{\beta, \bar{\alpha}}=\varnothing .
$$

Some more notation: write

$$
\Sigma(\mathrm{x}):=\left\{1 \leq k \leq \nu: x_{k-1} \in \mathcal{B}\left(\beta, \alpha_{k}\right)\right\}, \quad \sigma(\mathrm{x}):=\sharp \Sigma(\mathrm{x})
$$

for each $\mathrm{x}=\left(x_{0}, x_{1}, \ldots, x_{\nu} ; \overline{\boldsymbol{\alpha}}\right) \in O_{\overline{\boldsymbol{\alpha}}}$. Additionally, let $k_{1}<k_{2}<\cdots<k_{\sigma(\mathrm{x})}$ denote the ordering of the elements of $\Sigma(\mathrm{x})$. By (4.6), $\sigma(\mathrm{x}) \geq m$ for each $\mathrm{x} \in O_{\overline{\boldsymbol{\alpha}}}$. With these facts, we can estimate $\sharp O_{\overline{\boldsymbol{\alpha}}}$. To do so, pick and fix an $\mathrm{x}=\left(x_{0}, x_{1}, \ldots, x_{\nu} ; \overline{\boldsymbol{\alpha}}\right) \in O_{\overline{\boldsymbol{\alpha}}}$. By construction:

$$
\sharp S\left(x_{k}, k\right)= \begin{cases}1, & \text { if } k \notin \Sigma(\mathrm{x}), \\ d_{\alpha_{k}}, & \text { if } k \in \Sigma(\mathrm{x}) .\end{cases}
$$

This means that in $O_{\bar{\alpha}}$ :

(*) we can find $d_{\alpha_{k_{l}}}$ distinct $\nu$-orbits that traverse the points $x_{k_{l}}, x_{k_{l}+1}, \ldots, x_{\nu} \in$ $X$ corresponding, respectively, to iterations of orders $k_{l}, k_{l}+1, \ldots, \nu$ of $\Gamma_{\mathscr{G}}$, $l=1, \ldots \sigma(\mathrm{x})$.

This implies that $O_{\bar{\alpha}}$ would have the smallest possible number of orbits of the kind described by $(*)$ if $\nu_{1}+\cdots+\nu_{J}=: d(\overline{\boldsymbol{\alpha}})$ of the elements of $\Sigma(\mathrm{x})$ were to correspond to $\nu_{j}$ distinct terms in the tuple $\left(x_{0}, \ldots, x_{\nu-1}\right)$ being in $\mathcal{B}(\beta, j), j=1, \ldots, J$. From this discussion and $(*)$, we get the (perhaps very conservative) lower bound:

$$
n(\varepsilon(\beta), \overline{\boldsymbol{\alpha}}) \geq \sharp O_{\overline{\boldsymbol{\alpha}}} \geq d_{1}^{\nu_{1}} \ldots d_{J}^{\nu_{J}} d_{J+1}^{m-d(\overline{\boldsymbol{\alpha}})} .
$$

Here, $n(\varepsilon(\beta), \overline{\boldsymbol{\alpha}})$ is as in Lemma 4.1, and the first inequality in (4.7) is owing to the fact that $O_{\overline{\boldsymbol{\alpha}}}$ is $(\varepsilon(\beta), \overline{\boldsymbol{\alpha}})$-separated.

Now, given any $\left(\nu_{1}, \ldots, \nu_{N}\right) \in \mathbf{N}^{N}$ satisfying $\nu_{1}+\cdots+\nu_{N}=[\beta \nu]+1$, we can find an $\overline{\boldsymbol{\alpha}} \in\{1, \ldots, N\}^{\nu}$ so that the $\nu_{j}$ 's are related to this $\overline{\boldsymbol{\alpha}}$ precisely as in the last paragraph. Thus:

$$
\begin{aligned}
\sum_{\overline{\boldsymbol{\alpha}} \in\{1, \ldots, N\}^{\nu}} n(\varepsilon, \overline{\boldsymbol{\alpha}}) & \geq \sum_{\substack{\nu_{1}, \ldots, \nu_{N} \in \mathbf{N} \\
\nu_{1}+\cdots+\nu_{N}=m}} d_{1}^{\nu_{1}} \ldots d_{N}^{\nu_{N}} \\
& =\left(d_{1}+\cdots+d_{N}\right)^{m} \geq\left(d_{1}+\cdots+d_{N}\right)^{\beta \nu} .
\end{aligned}
$$


Applying Lemma 4.1, this gives

$$
h_{\text {top }}(S, \mathscr{G}):=h_{\text {top }}\left(\Gamma_{\mathscr{G}}\right) \geq \beta \log \left(d_{1}+\cdots+d_{N}\right) .
$$

However, as this holds for any $\beta \in(0,1)$, letting $\beta \rightarrow 1^{-}$, we get

$$
h(S, \mathscr{G}) \geq \log \left(d_{1}+\ldots d_{N}\right) .
$$

This establishes the lower bound in (4.1), and hence the result.

We remark here that it is, in general, not possible to get a cleaner upper bound for $h_{\text {top }}(S, \mathscr{G})$ than (4.1). For instance, there isn't, in general, a way to determine which of the numbers $\left\{d_{1}(f), \ldots, d_{n}(f)\right\}$ is the largest even for $f: X \longrightarrow X$ surjective and holomorphic (let alone for a general correspondence $\Gamma$ ). We shall not discuss here what is known in general about the function $\{0,1, \ldots, n\} \ni p \mapsto d_{p}(f)$. However, for $X$ as above, $\lambda_{p}(f)$, for $f: X \longrightarrow X$ holomorphic and $p=0,1, \ldots, n$, can be determined cohomologically. This can lead to cleaner expressions whenever $H^{p, p}(X ; \mathbf{R})$ are one-dimensional for each $p=1, \ldots, n$. This, essentially, is what underlies

The proof of Theorem 1.8. Russakovskii-Shiffman have shown [16, Section 4] that for any non-constant holomorphic map $f: \mathbf{P}^{n} \longrightarrow \mathbf{P}^{n}$

$$
\lambda_{p}(f)=d_{p}(f) \quad \text { and } \quad d_{p}(f)=d_{1}(f)^{p} \quad \text { for } p=1, \ldots, n .
$$

As argued in the proof of Theorem $4.2, d_{n}(f)=d_{\text {top }}(f)$. Thus, from the above facts, we get

$$
\lambda_{p}(f)=d_{\text {top }}(f)^{p / n}, \quad \text { for } p=1, \ldots, n .
$$

Fix a set of generators $\left\{f_{1}, \ldots, f_{N}\right\}$ of $S$. Clearly, by definition, $\lambda_{p}\left(\Gamma_{\mathscr{G}}^{\circ \nu}\right)$ is the sum of the $p^{\text {th }}$ intermediate degrees of the maps, counted according to multiplicity, whose graphs constitute $\Gamma_{\mathscr{G}}^{\circ \nu}$. From (4.9), we see that for $\mathbf{P}^{n}, \lambda_{p}, p=1, \ldots, n$, is multiplicative with respect to composition of non-constant holomorphic self-maps. Thus, by (4.8), we get

$$
\lambda_{p}\left(\Gamma_{\mathscr{G}}^{\circ \nu}\right)=\left(d_{1}\left(f_{1}\right)^{p}+\cdots+d_{1}\left(f_{N}\right)^{p}\right)^{\nu} \quad \text { for } p=1, \ldots, n .
$$

Hence, $d_{p}\left(\Gamma_{\mathscr{G}}\right)=\left(d_{1}\left(f_{1}\right)^{p}+\cdots+d_{1}\left(f_{N}\right)^{p}\right), p=1, \ldots, n$. As for $p=0: d_{0}\left(\Gamma_{\mathscr{G}}\right)=N$. Given these facts, the conclusion of Theorem 1.8 follows from Theorem 4.2.

Corollary 1.9 now follows immediately.

\section{Concerning Friedland's entropy}

This section is dedicated to the result on $h_{F}$ mentioned in Section $1-$ i.e., Theorem 5.2. First, however, we need some notation and a lemma. Let $S$ be a finitely generated rational semigroup on $\mathbf{P}^{1}$. If we fix a finite set of generators $\mathscr{G}$, then the space $\Gamma^{\infty}$ (introduced in Definition 1.2) corresponding to $\Gamma_{\mathscr{G}}$ will be denoted by $\Gamma_{\mathscr{G}}^{\infty}$. Also, we abbreviate ${ }^{\Gamma \mathscr{G}} \mathbf{P}^{1}$ to ${ }^{\mathscr{G}} \mathbf{P}^{1}$. Given any holomorphic correspondence $\Gamma$ from $X_{1}$ to $X_{2}$, where $X_{i}, i=1,2$, are as in Definition 1.1, we define

$$
F_{\Gamma}(x):=\pi_{2}\left(\left(\left.\pi_{1}\right|_{|\Gamma|}\right)^{-1}\{x\}\right) \quad \forall x \in X_{1},
$$

and write $F_{\Gamma}^{\nu}(x):=F_{\Gamma^{\circ \nu}}(x)$. Coming back to the correspondence $\Gamma_{\mathscr{G}}$ : we abbreviate $F_{\Gamma_{\mathscr{G}}}^{\nu}(x)$ to $F_{\mathscr{G}}^{\nu}(x)$. 
Lemma 5.1. Let $S$ be a finitely generated rational semigroup on $\mathbf{P}^{1}$. Let $\mathscr{G}=$ $\left\{f_{1}, \ldots, f_{N}\right\}$ be a set of generators of $S$. Write

$$
\mathfrak{S}_{\mathscr{G}}:=\bigcup_{j=1}^{N} \bigcup_{i \neq j}\left\{x \in \mathbf{P}^{1}: f_{i}(x)=f_{j}(x)\right\} .
$$

Consider a point $\mathscr{O}=\left(x_{0}, x_{1}, x_{2}, \ldots\right) \in \Gamma_{\mathscr{G}}^{\infty}$. If the pre-image of $\mathscr{O}$ under the map $\pi_{\text {itn }}:{ }^{\mathscr{G}} \mathbf{P}^{1} \longrightarrow \Gamma_{\mathscr{G}}^{\infty}$ is infinite, then there exist an $n^{\bullet} \in \mathbf{N}$ and a point

$$
x^{\bullet} \in \mathfrak{S}_{\mathscr{G}} \bigcap\left(\limsup _{\nu \rightarrow \infty} F_{\mathscr{G}}^{\nu}\left(x^{\bullet}\right)\right)
$$

such that $x^{\bullet}=x_{n} \bullet$.

Proof. First consider any $\mathscr{O}=\left(x_{0}, x_{1}, x_{2}, \ldots\right) \in \Gamma_{\mathscr{G}}^{\infty}$. For each $\nu \in \mathbf{Z}_{+}$, there are only finitely many $j$ such that $f_{j}\left(x_{\nu-1}\right)=x_{\nu}$. Thus, it is easy to see that $\pi_{\text {itn }}^{-1}\{\mathscr{O}\}$ is infinite if and only if

$(* *)$ there exists a sequence of positive integers $\nu_{1}<\nu_{2}<\nu_{3}<\cdots$ such that for each $k \in \mathbf{Z}_{+}, f_{j}\left(x_{\left(\nu_{k}-1\right)}\right)=x_{\nu_{k}}$ for more than one $j \in\{1, \ldots, N\}$.

Now, assume that $\pi_{\mathrm{itn}}^{-1}\{\mathscr{O}\}$ is infinite. Then, by $(* *)$ there exists a sequence of positive integers $\nu_{1}<\nu_{2}<\nu_{3}<\cdots$ such that

$$
x_{\left(\nu_{k}-1\right)} \in \mathfrak{S}_{\mathscr{G}} \quad \forall k \in \mathbf{Z}_{+} .
$$

Since $f_{1}, \ldots, f_{N}$ are distinct and $\mathbf{P}^{1}$ is one-dimensional, $\mathfrak{S}_{\mathscr{G}}$ is finite. Thus, by (5.1), we conclude that there exists an increasing subsequence $\left\{\nu_{k_{\ell}}\right\} \subset\left\{\nu_{k}\right\}$ and a point $x^{\bullet} \in \mathfrak{S}_{\mathscr{G}}$ such that

$$
x_{\left(\nu_{k_{\ell}}-1\right)}=x^{\bullet} \in \mathfrak{S}_{\mathscr{G}} \quad \forall \ell \in \mathbf{Z}_{+} .
$$

If we write $n_{\ell}:=\nu_{k_{\ell}}-\nu_{k_{1}}$, then the above equation implies that $x^{\bullet} \in F_{\mathscr{G}}^{n_{\ell}}\left(x^{\bullet}\right)$ for every $\ell \in \mathbf{Z}_{+} \backslash\{1\}$. Therefore, we conclude that

$$
x^{\bullet} \in \bigcap_{k \in \mathbf{N}} \bigcup_{\nu \geq k} F_{\mathscr{G}}^{\nu}\left(x^{\bullet}\right)=: \limsup _{\nu \rightarrow \infty} F_{\mathscr{G}}^{\nu}\left(x^{\bullet}\right) .
$$

Taking $n^{\bullet}=\left(\nu_{k_{1}}-1\right)$, the desired conclusion is obtained.

Before we present Theorem 5.2, we elaborate upon the remark made towards the end of Section 1. For the purposes of this discussion, let $X$ be any compact metric space and let $S$ be the semigroup generated by the maps $f_{j}: X \longrightarrow X, j=1, \ldots, N$, that are continuous, and take $\Gamma=\cup_{1 \leq j \leq N} \operatorname{graph}\left(f_{j}\right)$ in Definition 1.2. For each $A \varsubsetneqq\{1, \ldots, N\}, A \neq \varnothing$, consider

$$
Y^{(A)}:=\left\{\left(x_{0}, x_{1}, x_{2}, \ldots\right) \in \Gamma^{\infty}: x_{n+1}=f_{j}\left(x_{n}\right) \text { for some } j \in A, n=0,1,2, \ldots\right\} .
$$

$Y^{(A)}$ is a closed subspace of $\Gamma^{\infty}$ that is invariant under $\sigma$, where $\sigma$ is as in Definition 1.2. Recalling the definition of $h_{F}(S, \mathscr{G})$, the basic properties of Bowen's entropy, and as $Y^{(A)}$ is $\sigma$-invariant, we get (as before, $\mathscr{G}:=\left\{f_{1}, \ldots, f_{N}\right\}$ )

$$
h_{F}(S, \mathscr{G}) \geq h\left(\left.\sigma\right|_{Y^{(A)}}\right)=h_{F}\left(\left\langle f_{j}: j \in A\right\rangle,\left\{f_{j}: j \in A\right\}\right) .
$$

When $A=\{j\}$, write $Y^{(A)}=: Y^{(j)}$. Observe: $f_{j}$ is conjugate to $\left.\sigma\right|_{Y^{(j)}}$ via the map $x \longmapsto\left(x, f_{j}(x), f_{j} \circ f_{j}(x), \ldots\right) \in Y^{(j)}$. From this and our preceding argument, we get

$$
h_{F}(S, \mathscr{G}) \geq h\left(\left.\sigma\right|_{Y^{(j)}}\right)=h\left(f_{j}\right) \quad \forall j=1, \ldots, N .
$$

Hence, $h_{F}(S, \mathscr{G}) \geq \max _{1 \leq j \leq N} h\left(f_{j}\right)$. In particular, this estimate holds true for all the semigroups discussed in Sections 1, 4 and the present section.

One might intuit from the previous lemma that the latter lower bounds could be improved (as least when $S$ is a finitely generated rational semigroup on $\mathbf{P}^{1}$ ). That 
intuition motivates the principal result of this section. We follow below the notation established for Proposition 3.5-for instance, $\mathscr{S}$ is the shift map introduced by that proposition.

Theorem 5.2. Let $S$ be a finitely generated rational semigroup on $\mathbf{P}^{1}$. Let $\mathscr{G}=\left\{f_{1}, \ldots, f_{N}\right\}$ be a set of generators of $S$. Define

$$
\mathcal{E}(\mathscr{G}):=\left\{\left(x_{0}, x_{1}, x_{2}, \ldots\right) \in \Gamma_{\mathscr{G}}^{\infty}: x_{0} \in \mathfrak{S}_{\mathscr{G}} \text { and } x_{0} \in \limsup _{\nu \rightarrow \infty} F_{\mathscr{G}}^{\nu}\left(x_{0}\right)\right\} .
$$

Then, Friedland's entropy satisfies

$$
\begin{aligned}
\log \left(\sum_{1 \leq j \leq N} \operatorname{deg}\left(f_{j}\right)\right)-\sup _{\mathscr{O} \in \mathcal{E}(\mathscr{G})} h\left(\mathscr{S}, \pi_{\text {itn }}^{-1}\{\mathscr{O}\}\right) & \leq h_{F}(S, \mathscr{G}) \\
& \leq \log \left(\sum_{1 \leq j \leq N} \operatorname{deg}\left(f_{j}\right)\right) .
\end{aligned}
$$

Before seeing a proof of the above theorem, it might be helpful to discuss when the estimates (5.2) are informative. To this end, we refer to the paragraph just after the proof of Lemma 5.1. We see there a lower bound that applies to any finitely generated rational semigroup on $\mathbf{P}^{1}$. To the best of our knowledge, this (apart from the exact computations for semigroups of Möbius transformations in [8]) is the only lower bound known for $h_{F}(S, \mathscr{G})$. With this in mind: an example of a class of $(S, \mathscr{G})$ for which $(5.2)$ would be informative is a class comprising finitely generated semigroups of Möbius transformations with $\sharp \mathscr{G} \geq 3$ such that the left-hand side of (5.2) is positive (however small). One way to achieve this, for $(S, \mathscr{G})$ as described, is for the maps in each proper subset of $\mathscr{G}$ of cardinality $(\sharp \mathscr{G}-1)$ to have common fixed points (along with a technical condition) but for the maps in $\mathscr{G}$ to have no fixed points in common. This endows the elements in $\mathcal{E}(\mathscr{G})$ with a very specific structure. However, since

- a detailed discussion of such an example would be rather protracted, and

- we do not see an overarching geometric description for (the class of) such semigroups,

we shall not dwell any further on this. That being said, there is an alternative approach that involves establishing that $\left({ }^{\mathscr{G}} \mathbf{P}^{1}, \mu\right)$ is isomorphic to $\left(\Gamma_{\mathscr{G}}^{\infty},\left(\pi_{\mathrm{itn}}\right)_{*} \mu\right)$ for an appropriate Markov measure $\mu$, which accounts more coherently for the $(S, \mathscr{G}$ )'s alluded to above. This is currently work in progress by the first-named author; details will appear elsewhere.

We present an example where the lower bound in (5.2) is informative for a different reason, and in which we get to see some of the reasoning hinted at above. Consider the semigroup $S$ whose set of generators $\mathscr{G}$ comprises the two functions $f_{1}$ and $f_{2}$ such that

$$
\left.f_{j}\right|_{\mathbf{C}}: z \longmapsto z+a_{j}, \quad j=1,2,
$$

where $a_{1} \neq a_{2} \in \mathbf{C}$. So, $\mathfrak{S}_{\mathscr{G}}=\{\infty\}$. Moreover, as $\infty$ is the common fixed point of $f_{1}$ and $f_{2}, \mathcal{E}(\mathscr{G})$ is a singleton consisting of the constant sequence $\mathscr{O}_{\infty}=(\infty, \infty, \ldots)$. Thus

$$
\pi_{\text {itn }}^{-1}\left\{\mathscr{O}_{\infty}\right\}=\left\{\left(\infty, \infty, \infty \ldots ; \alpha_{1}, \alpha_{2}, \ldots\right): \alpha_{j} \in\{1,2\} \text { for each } j \in \mathbf{Z}_{+}\right\} .
$$

Observe that $\mathscr{S}\left(\pi_{\text {itn }}^{-1}\left\{\mathscr{O}_{\infty}\right\}\right) \subseteq \pi_{\text {itn }}^{-1}\left\{\mathscr{O}_{\infty}\right\}$. Thus, the iterated application of $\mathscr{S}$ on $\pi_{\text {itn }}^{-1}\left\{\mathscr{O}_{\infty}\right\}$ behaves like the dynamics of the left-shift on $\{1,2\}^{\mathbf{N}}$, whence we get

$$
h\left(\mathscr{S}, \pi_{\text {itn }}^{-1}\left\{\mathscr{O}_{\infty}\right\}\right)=\log (2) .
$$


Thus, (5.2) gives 0 as the lower bound for $h_{F}(S, \mathscr{G})$. However, there is no better lower bound for $h_{F}(S, \mathscr{G})$ in the vast majority of the examples in this class! Specifically, if $\left\{a_{1}, a_{2}\right\}$ is linearly independent over $\mathbf{R}$, or if $a_{2}=c a_{2}$ for some $c \geq 0$, then $h_{F}(S, \mathscr{G})=0$ - see [8, Lemma 5.3]. With those comments on (5.2), we now present

The proof of Theorem 5.2. Consider the compact metric spaces ${ }^{\mathscr{C}} \mathbf{P}^{1}$ and $\Gamma_{\mathscr{G}}^{\infty}$. Denote by $\bar{\sigma}: \Gamma_{\mathscr{G}}^{\infty} \longrightarrow \Gamma_{\mathscr{G}}^{\infty}$ the shift

$$
\Gamma_{\mathscr{G}}^{\infty} \ni\left(x_{0}, x_{1}, x_{2}, \ldots\right) \mapsto\left(x_{1}, x_{2}, x_{3}, \ldots\right) .
$$

Recall that $\mathscr{S}:{ }^{\mathscr{G}} \mathrm{X} \longrightarrow{ }^{\mathscr{G}} \mathrm{X}$ is as described in Proposition 3.5. We have:

- $\pi_{\text {itn }}$ is a continuous surjective map; and

- $\pi_{\text {itn }} \circ \mathscr{S}=\bar{\sigma} \circ \pi_{\text {itn }}$.

In other words, $\bar{\sigma}$ is a factor of $\mathscr{S}$. Thus, Proposition 3.5 and Corollary 1.9 together imply:

$$
h_{F}(S, \mathscr{G}):=h(\bar{\sigma}) \leq h(\mathscr{S})=h_{\mathrm{top}}(S, \mathscr{G})=\log \left(\sum_{1 \leq j \leq N} \operatorname{deg}\left(f_{j}\right)\right) .
$$

We now derive the lower bound for $h_{F}$. For the moment, fix an $\mathscr{O} \in \Gamma_{\mathscr{G}}^{\infty}$. We have two cases.

Case 1. $\pi_{\text {itn }}^{-1}\{\mathscr{O}\}$ is a finite set. For any $\nu \in \mathbf{Z}_{+}$and $\varepsilon>0, \mathscr{O}(\varepsilon, \nu)$-spans itself. Thus, the finiteness of $\pi_{\text {itn }}^{-1}\{\mathscr{O}\}$ implies that $h\left(\mathscr{S}, \pi_{\text {itn }}^{-1}\{\mathscr{O}\}\right)=0$.

Case 2. $\pi_{\text {itn }}^{-1}\{\mathscr{O}\}$ is an infinite set. Write $\mathscr{O}=\left(x_{0}, x_{1}, x_{2}, \ldots\right)$. In this case, Lemma 5.1 enables us to define

$$
k(\mathscr{O}):=\min \left\{n \in \mathbf{N}: x_{n} \in \mathfrak{S}_{\mathscr{G}} \cap\left(\limsup _{\nu \rightarrow \infty} F_{\mathscr{G}}^{\nu}\left(x_{n}\right)\right)\right\} .
$$

If $k(\mathscr{O}) \geq 1$, then by definition, there is a fixed tuple $\left(\alpha_{1}, \ldots, \alpha_{k(\mathscr{O})}\right)$ such that every element of $\pi_{\text {itn }}^{-1}\{\mathscr{O}\}$ has the form $\left(x_{0}, \ldots, x_{k(\mathscr{O})}, \ldots ; \alpha_{1}, \ldots, \alpha_{k(\mathscr{O})}, \ldots\right)$. Thus, following the notation of the discussion that precedes Result 3.6, for each $\varepsilon>0$ we have:

$$
r_{\nu}\left(\varepsilon, \pi_{\text {itn }}^{-1}\{\mathscr{O}\}\right)=r_{\nu}\left(\varepsilon, \pi_{\text {itn }}^{-1}\left\{\left(x_{k(\mathscr{O})}, x_{k(\mathscr{O})+1}, x_{k(\mathscr{O})+2}, \ldots\right)\right\}\right) \quad \forall \nu \text { suffiiciently large. }
$$

We therefore conclude (irrespective of whether $k(\mathscr{O})=0$ or $k(\mathscr{O}) \geq 1$ ) that

$$
\begin{aligned}
h\left(\mathscr{S}, \pi_{\mathrm{itn}}^{-1}\{\mathscr{O}\}\right) & =h\left(\mathscr{S}, \pi_{\mathrm{itn}}^{-1}\left\{\left(x_{k(\mathscr{O})}, x_{k(\mathscr{O})+1}, x_{k(\mathscr{O})+2}, \ldots\right)\right\}\right), \text { and } \\
x_{k(\mathscr{O})} & \in \mathfrak{S}_{\mathscr{G}} \cap\left(\limsup _{\nu \rightarrow \infty} F_{\mathscr{G}}^{\nu}\left(x_{k(\mathscr{O})}\right)\right) .
\end{aligned}
$$

From the discussion of each of the above cases, and by (5.4), we get

$$
\sup _{\mathscr{O} \in \Gamma_{\mathscr{G}}^{\infty}} h\left(\mathscr{S}, \pi_{\mathrm{itn}}^{-1}\{\mathscr{O}\}\right)=\sup _{\mathscr{O} \in \mathcal{E}(\mathscr{G})} h\left(\mathscr{S}, \pi_{\mathrm{itn}}^{-1}\{\mathscr{O}\}\right) .
$$

From this, Result 3.6 and Proposition 3.5, we have

$$
\begin{aligned}
h_{\mathrm{top}}(S, \mathscr{G})=h(\mathscr{S}) & \leq h(\bar{\sigma})+\sup _{\mathscr{O} \in \mathcal{E}(\mathscr{G})} h\left(\mathscr{S}, \pi_{\mathrm{itn}}^{-1}\{\mathscr{O}\}\right) \\
& =h_{F}(S, \mathscr{G})+\sup _{\mathscr{O} \in \mathcal{E}(\mathscr{G})} h\left(\mathscr{S}, \pi_{\mathrm{itn}}^{-1}\{\mathscr{O}\}\right) .
\end{aligned}
$$

From (5.3) and (5.5), and given the conclusion of Corollary 1.9, the theorem follows.

Remark 5.3. Theorem 5.2 can be extended to higher dimensions. But, in that case, it is possible for the set $\mathfrak{S}_{\mathscr{G}}$ introduced in Lemma 5.1 to contain algebraic varieties of positive dimension. In view of the discussion preceding the proof of Theorem 5.2, it seems likely that it would be difficult to understand the analogue of 
the lower bound of $h_{F}(S, \mathscr{G})$ in higher dimensions. Therefore, we have focused on classical rational semigroups in this section.

Acknowledgments. Gautam Bharali is supported by a Swarnajayanti Fellowship (Grant no. DST/SJF/MSA-02/2013-14) and a UGC CAS-II grant (Grant No. F.510/25/CAS-II/2018(SAP-I)).

\section{References}

[1] Bowen, R.: Entropy for group endomorphisms and homogeneous spaces. - Trans. Amer. Math. Soc. 153, 1971, 401-414.

[2] Bullett, S., and M. Freiberger: Holomorphic correspondences mating Chebyshev-like maps with Hecke groups. - Ergodic Theory Dynam. Systems 25:4, 2005, 1057-1090.

[3] Bullett, S., and C. Penrose: Mating quadratic maps with the modular group. - Invent. Math. 115:3, 1994, 483-511.

[4] Dinh, T.-C., and N. Sibony: Distribution des valeurs de transformations méromorphes et applications. - Comment. Math. Helv. 81:1, 2006, 221-258.

[5] Dinh, T.-C., and N. SiBony: Upper bound for the topological entropy of a meromorphic correspondence. - Israel J. Math. 163, 2008, 29-44.

[6] Fornaess, J. E., and E. Mihailescu: Equilibrium measures on saddle sets of holomorphic maps on $\mathbf{P}^{2}$. - Math. Ann. 356:4, 2013, 1471-1491.

[7] Friedland, S.: Entropy of algebraic maps. - In: Proceedings of the Conference in Honor of Jean-Pierre Kahane (Orsay, 1993), J. Fourier Anal. Appl. Special Issue, 1995, 215-228.

[8] Friedland, S.: Entropy of graphs, semigroups and groups. - In: Ergodic Theory of $\mathbf{Z}^{d}$ Actions (Warwick, 1993-1994), London Math. Soc. Lecture Note Ser. 228, Cambridge Univ. Press, Cambridge, 1996, 319-343.

[9] Geller, W., and M. Pollicott: An entropy for $\mathbf{Z}^{2}$-actions with finite entropy generators. - Fund. Math. 157:2-3, 1998, 209-220.

[10] Ghys, É., R. Langevin, and P. Walczak: Entropie géométrique des feuilletages. - Acta Math. 160:1-2, 1988, 105-142.

[11] Gromov, M.: On the entropy of holomorphic maps. - Enseign. Math. 49:3-4, 2003, 217-235.

[12] Hinkkanen, A., and G. J. Martin: The dynamics of semigroups of rational functions I. Proc. London Math. Soc. 73:2, 1996, 358-384.

[13] Katok, A., and B. Hasselblatt: Introduction to the modern theory of dynamical systems. - Encyclopedia Math. Appl. 54, Cambridge Univ. Press, Cambridge, 1995.

[14] Mihailescu, E., and M. URBański: Inverse topological pressure with applications to holomorphic dynamics of several complex variables. - Commun. Contemp. Math. 6:4, 2004, 653-679.

[15] Misiurewicz, M., and F. Przytycki: Topological entropy and degree of smooth mappings. - Bull. Acad. Polon. Sci. Sér. Sci. Math. Astronom. Phys. 25:6, 1977, 573-574.

[16] Russakovskit, A., and B. Schiffman: Value distribution for sequences of rational mappings and complex dynamics. - Indiana Univ. Math. J. 46, 1997, 897-932.

Received 29 April $2020 \bullet$ Revised received 7 April $2021 \bullet$ Accepted 4 May 2021 